\title{
Im Aufbau
}

VON GABRIELE MOOS,

UTA ROTHERMEL, MATTHIAS

KONRAD, KLAUS TITZ

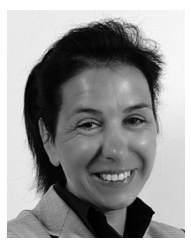

Prof. Dr. Gabriele Moos ist Hochschullehrerin für Sozialmanagement am RheinAhrCampus in Remagen und leitet dort den Studiengang Gesundheits- und Sozialwirtschaft.

www.hs-koblenz.de

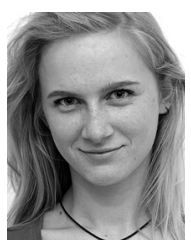

Uta Rothermel hat Soziale Arbeit und Philosophie studiert und arbeitet als Projektassistenz bei der contec $\mathrm{GmbH}$. www.contec.de

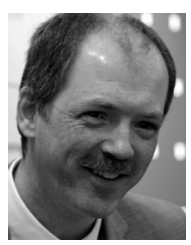

Matthias Konrad ist Mitglied der Geschäftsleitung der contec $\mathrm{GmbH}$ und leitet das Marktfeld der

Behindertenhilfe. www.contec.de

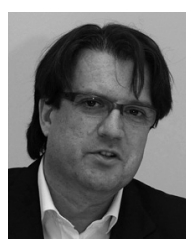

Klaus Titz arbeitet als wissenschaftlicher Referent im Deutscher Verein für öffentliche und private Fürsorge im Arbeitsfeld »Alter, Pflege, Rehabilitation, Gesundheit, Steuerung sozialer Dienste«. www.deutscher-verein.de

\section{Eine neue Studie zeigt: Das Controlling spielt in vielen kommunalen Jugend- und Sozialhilfeverwaltungen zwar bereits eine gewichtige Rolle, ist aber durchaus noch ausbaufähig.}

Im Jahr 2011 veröffentlichte die contec $\mathrm{GmbH}$ in Zusammenarbeit mit der Deutschen Gesellschaft für Management und Controlling in der Sozialwirtschaft e.V. (DGSC) die Studie »Controlling in der Sozialwirtschaft - Ausbaustand und Perspektiven «. In deren Rahmen wurden Leistungsanbieter der Sozialwirtschaft zum Stand ihres Controllings befragt.

Auf der Grundlage dieser Studie wurde in 2013 eine Studie zu Controlling in kommunalen Jugend- und Sozialhilfeverwaltungen veröffentlicht. Ziel der Kooperation des Rhein AhrCampus, der contec $\mathrm{GmbH}$ und des Deutschen Vereins für öffentliche und private Fürsorge e. V. war, den Ausbaustand von Controlling in der kommunalen Jugend- und Sozialhilfe zu untersuchen. Anhand eines Fragebogens wurden die Fachbereichsund Dezernatsleitungen aller Kreise und kreisfreien Städte befragt.

Zunächst wurden allgemeine Fragen zur Organisationsstruktur und zur Institutionalisierung von Controlling als ganzem gestellt, an die sich speziellere Fragen zum operativen-, strategischenund Wirkungscontrolling anschlossen. Im Folgenden werden einige zentrale Ergebnisse der Untersuchung zusammengefasst.

\section{Strategisches Controlling}

Das strategische Controlling bezieht sich auf die Zielentwicklung, Aufgabenentwicklung und Ergebniskontrolle von mittel- und langfristigen Entwicklungszielen. Dabei unterstützt das Controlling den Zielentwicklungs- und Entscheidungsprozess der Leitungsebene.
Die Schlüsselfrage für das strategische Controlling lautet: Tun wir die richtigen Dinge? Für das strategische Controlling wurden das Vorhandensein und die Umsetzung von Strategie und Leitbild sowie der Ausbaustand von Instrumenten der Analyse und Steuerung erhoben.

Etwas mehr als die Hälfte Teilnehmenden gaben an, dass bei ihnen eine Strategie vorhanden ist, bei fast einem Viertel ist eine Strategie in Entwicklung und bei knapp unter einem Fünftel ist keine Strategie vorhanden. Bezogen auf ein Leitbild gaben ähnlich viele der Teilnehmenden an, dass ein Leitbild vorhanden ist, ein Leitbild ist jedoch nur bei wenigen in Entwicklung und sogar bei fast einem Drittel der Fachbereiche nicht vorhanden (vgl. Abb. 1).

Interessant wäre an dieser Stelle eine tiefer gehende Analyse zur Bedeutung der Strategie für die Arbeit der jeweiligen Verwaltung. Sind Leitbilder und Strategien tatsächlich auf der operativen Ebenen handlungsleitend oder werden sie allein auf höheren Leitungsebenen als Handlungsgrundlage verwendet? Ein Indiz dafür, dass Strategien nicht umfassend verankert sind, ist, dass nur knapp ein Fünftel der antwortenden Kommunen ihre Budgetplanung an strategischen Zielen orientieren, obwohl weit mehr angeben, über eine Strategie zu verfügen.

Die vorhanden Leitbilder oder Strategien sollten regelmäßig auf ihre Gültigkeit überprüft und gegebenfalls aktualisiert werden. Erhoben wurde daher, ob und wie regelmäßig in den Fachbereichen eine Überprüfung und Aktualisierung der Strategie stattfindet. 


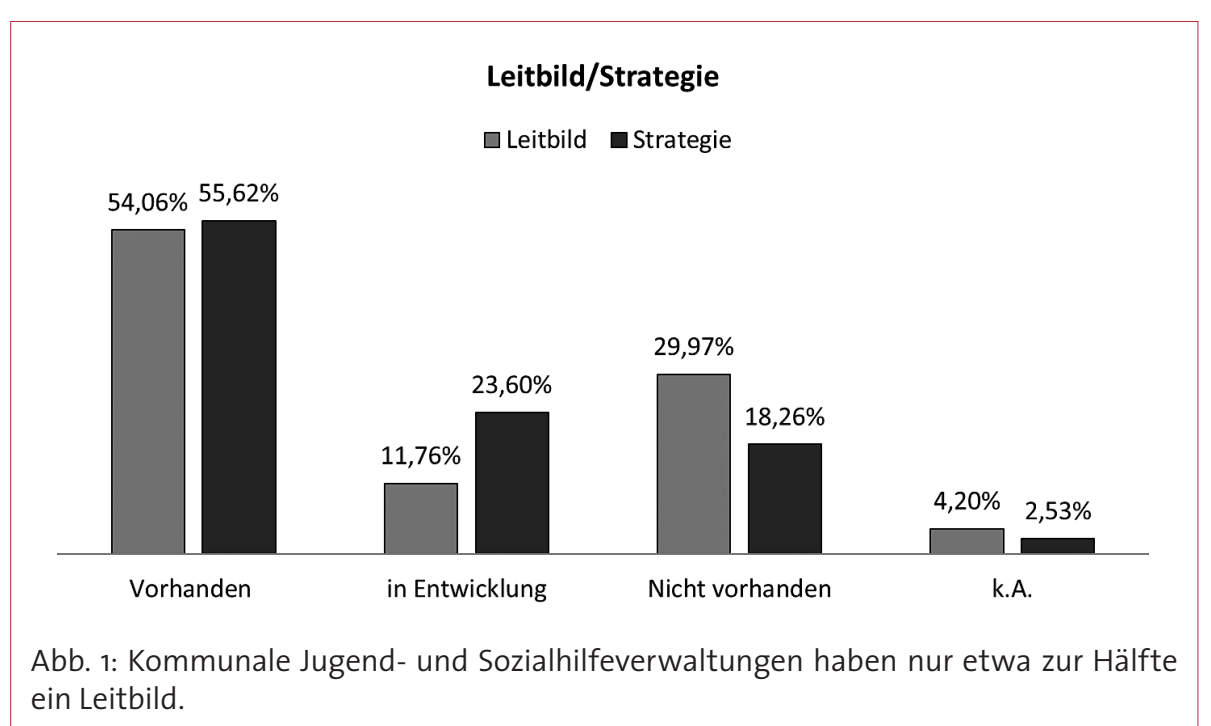

Fast ein Viertel gaben auf die Frage nach der Überprüfung und Aktualisierung von Strategie und Leitbild keine Antwort, es gaben jedoch nur einzelne an, dass Strategie und Leitbild bei ihnen nie überprüft und aktualisiert würden. Am häufigsten wird eine Strategieüberprüfung anlassbezogen umgesetzt. Etwa jeweils ein Fünftel der Fachbereiche antworteten mit »in unregelmäßigen Abständen " oder gaben an, dass die Strategie oder das Leitbild regelmäßig in einem bestimmten zeitlichen Intervall überprüft wird.

Eine strategieorientierte Arbeit scheint für den Teil der Kommunen von besonderer Relevanz zu sein, die in regelmäßigen Abständen die Strategie überprüfen. Eine anlassbezogene Strategieüberprüfung könnte beispielsweise durch Wechsel der politischen Rahmenbedingungen, durch massive Veränderung der sozialen und wirtschaftlichen Lage oder durch gesetzliche Vorgaben ausgelöst werden.

Eine regelmäßige Überprüfung bedeutet, dass bei etwa einem Viertel mindestens quartalsweise, bei einigen mindestens halbjährlich und bei dem überwiegenden Anteil mindestens jährlich überprüft und aktualisiert wird.

\section{Instrumente strategischer Analyse und Steuerung}

Im Hinblick auf die langfristige Steuerung im Controlling wurde das Vorhandensein strategischer Analyseinstrumente erhoben (vgl. Abb. 2). Ergebnis: Abgesehen von Vergleichsringen ist der Anteil der Fachbereiche zu vernachlässigen, in denen eines oder mehrere der Analyseinstrumente vorhanden sind. In den Fach- bereichen Jugend und Soziales sind die genannten Instrumente bei weniger als einem Fünftel auch nur wenig vorhanden.

In Verbindung mit den Ergebnissen zur Strategie ist dieses Ergebnis keine Überraschung. Wesentlich für die Steuerung der Sozial- und Jugendhilfe sind Informationen über den Haushalt, Leistungsmengen und Leistungskosten. Aussagen darüber stellt in der Regel das Berichtswesen zur Verfügung. Die Informationen daraus können für die Verwendung in Vergleichsringen aufbereitet werden.

Die anderen Instrumente sind auf der strategischen Ebene angesiedelt und dürften auch deshalb keine breite An- wendung finden, weil strategiegeleitetes Handeln in den Anfängen zu stecken scheint oder nur begrenzt als bedeutende Stellschraube für die Wirksamkeit der Verwaltung gesehen wird.

In Verbindung mit der »Neuen Steuerung " auf die strategische Steuerung bezogene Erwartungen haben sich nicht erfüllt. Man könnte in dem Zusammenhang danach fragen, ob die Sozial- und Jugendhilfe überhaupt strategisch steuerbar ist oder sie so umfänglich von durch sie unbeeinflussten externen Faktoren abhängt, dass eigene Strategien nicht erfolgversprechend erscheinen.

Die Befragung zu strategischen Steuerungsinstrumenten ergibt analog dazu, dass diese von geringer Relevanz sind. Die Ergebnisse verstärken den Eindruck, dass sich das Handeln der Befragten vorwiegend an vergangenheitsorientierten Zahlen ausrichtet. Es ist festzustellen, dass weniger als 50 Prozent der befragten Kommunen über Ziele steuern.

Ohne Ziele fehlt dem Controlling die Grundlage, um zukunftsorientierte Steuerungsunterstützung zu leisten.

\section{"Das Handeln von Jugend- und Sozialämtern orientiert sich stark an Zahlen der Vergangenheit"}

Controlling bleibt bei der Beschreibung des Ist-Zustandes und der Darstellung von Zahlen ohne ergebnisbezogene, qualitative Aussagen. Damit bleibt es hinter seinen Möglichkeiten zurück, die Planung, Steuerung und Kontrolle des Managements zu unterstützen. Im

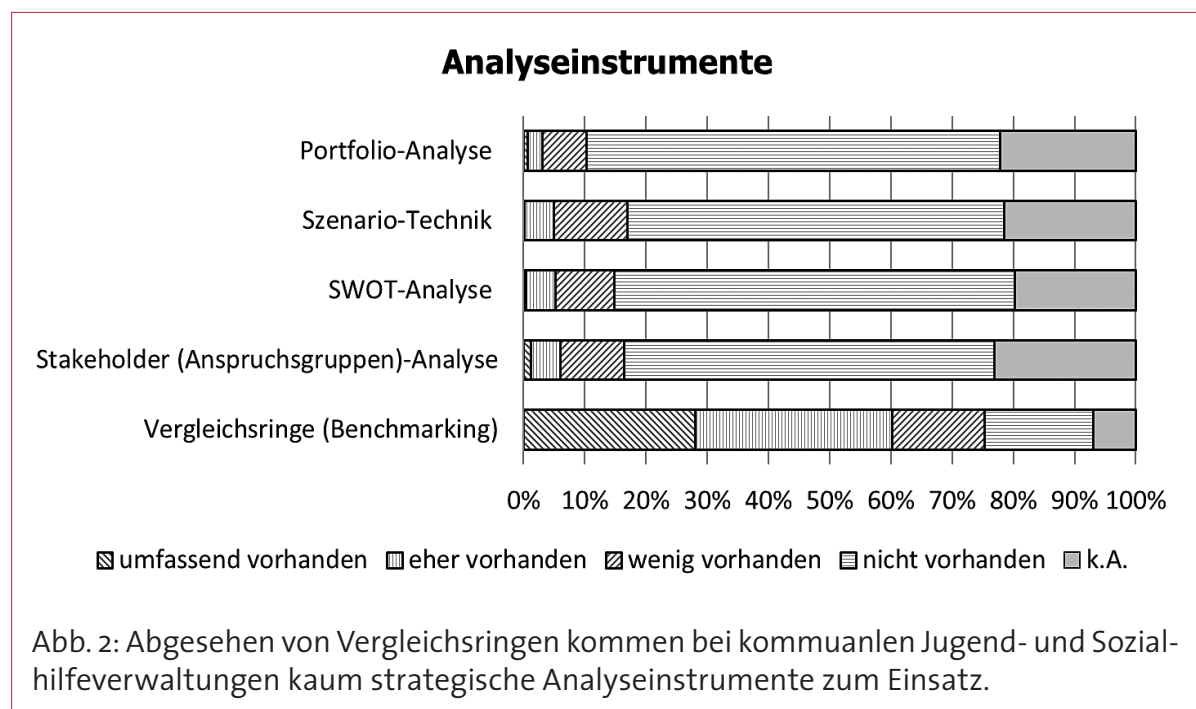

Abb. 2: Abgesehen von Vergleichsringen kommen bei kommuanlen Jugend- und Sozialhilfeverwaltungen kaum strategische Analyseinstrumente zum Einsatz. 
Bereich steuernder Systemeingriffe sind ziel- und zukunftsorientierte Ansätze (z. B. Balanced Scorecard) eher eine Ausnahme. Dies kann darauf zurückzuführen sein, dass Leistungsziele nur schwer $\mathrm{zu}$ operationalisieren sind.

\section{Wirkungsziele nur im Ansatz vorhanden}

Für die meisten Fachbereiche spielt Controlling eine mindestens wichtige Rolle für den Erfolg des Fachbereiches. Davon etwa die Hälfte schätzen Controlling sogar als sehr wichtig ein.

Obwohl Controlling in der Sozial- und Jugendhilfe über eine kurze Geschichte verfügt, ist man von dessen Bedeutung für den Organisationserfolg überzeugt. Ausgangspunkt für eine wirk- und damit erfolgsorientierte Steuerung ist die Zieldefinition.

Nur in vereinzelten Fachbereichen sind Wirkziele umfassend vorhanden, bei einem kleinen Teil sind Wirkziele immerhin im Wesentlichen vorhanden. In der Hälfte der Fachbereiche sind Wirkungsziele gerade einmal in Ansätzen vorhanden und bei etwa einem Viertel der Fachbereiche sind keine Wirkziele vorhanden. Insgesamt sind mindestens im Ansatz Wirkziele vorhanden (vgl. Abb. 3).

Das Vorhandensein von Wirkzielen in den Fachbereichen Jugend und Soziales unterscheidet sich deutlich. Während in der Sozialhilfe bei fast einem Drittel der Fachbereiche keine Wirkziele vorhanden sind, sind in der Jugendhilfe bei mehr als vier Fünftel Wirkziele vorhanden.

\section{Wirkungsmessung rudimentär}

Mit Verfahren der Wirkungsmessung wird überprüft, inwieweit Wirkziele erreicht werden. Dazu zählt beispielsweise der die Methode des »Social Return on Investment (SROI) «, der den gesellschaftlichen Nutzen sozialer Leistungen monetär messbar macht. Die Wirkungsmessung über den SROI wird bei lediglich zehn Prozent der Einrichtungen angewandt. Neben dem allgemeinen Problem, dass ohne Wirkziele keine Wirkungsmessung sinnvoll ist, kann dies daran liegen das die Erfassung des SROI sehr aufwendig und meist nur mit externer Begleitung zu erheben ist.

Vergleiche mit anderen Fachbereichen über Benchmarks sind eine Möglich-

\section{Wirkungsziele}

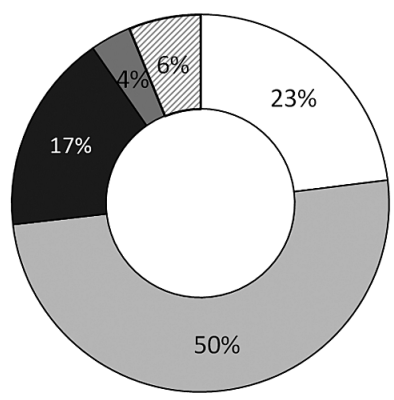

$\square$ nicht vorhanden

$\square$ in Ansätzen vorhanden

- im Wesentlichen vorhanden

$\square$ umfassend vorhanden

$\square$ keine Antwort

Abb. 3: Nur wenige der befragten kommunalen Jugend- und Sozialhilfeverwaltungen arbeiten mit umfassenden Wirkungszielen. keit, die eigenen Leistungen einzuordnen. Dieses Verfahren wird bei über 70 Prozent der Einrichtungen angewandt. Dies lässt sich damit erklären, dass ein solches Verfahren einfach umsetzbar ist und interne Vergleichszahlen genutzt werden können.

Auch über ein Qualitätsmanagementsystem können Informationen über Leistungen gewonnen werden. Die Qualität sozialer Leistungen misst sich nicht zuletzt an der Zufriedenheit der Leistungsempfänger. Nutzwert-Analysen ermöglichen fundierte Entscheidungen zwischen unterschiedlichen oder auch ähnlichen Leistungen. Kosten-Wirksamkeitsanalysen ermöglichen die Bewertung von Investitionen, deren Nutzen nicht monetär messbar ist und die in erster Linie eine qualitative Wirkung erzielen. Solche Verfahren sind in Ansätzen und nicht flächendeckend vorhanden.
Es kann festgestellt werden, dass Verfahren zur Wirkmessung in Jugendund Sozialhilfeverwaltungen wenig bis von Benchmarkingverfahren sind alle genannten Verfahren bei mehr als vier Fünfteln der Teilnehmenden höchstens wenig vorhanden (vgl. Abb. 4). Das der schweren Anwendbarkeit und des Mangels an Wirkungszielen bisher nicht verankert.

\section{Gespaltenes Bild beim Fachcontrolling}

Das Fachcontrolling bildet die Steuerung der Leistungserbringung selbst ab. Auffallend unterscheiden sich in diesem Punkt die Fachbereiche Jugend und Soziales. Über 90 Prozent in der Jugendhilfe haben Fachcontrolling, während in der nicht vorhanden sind. Mit Ausnahme Wirkungscontrolling ist somit aufgrund

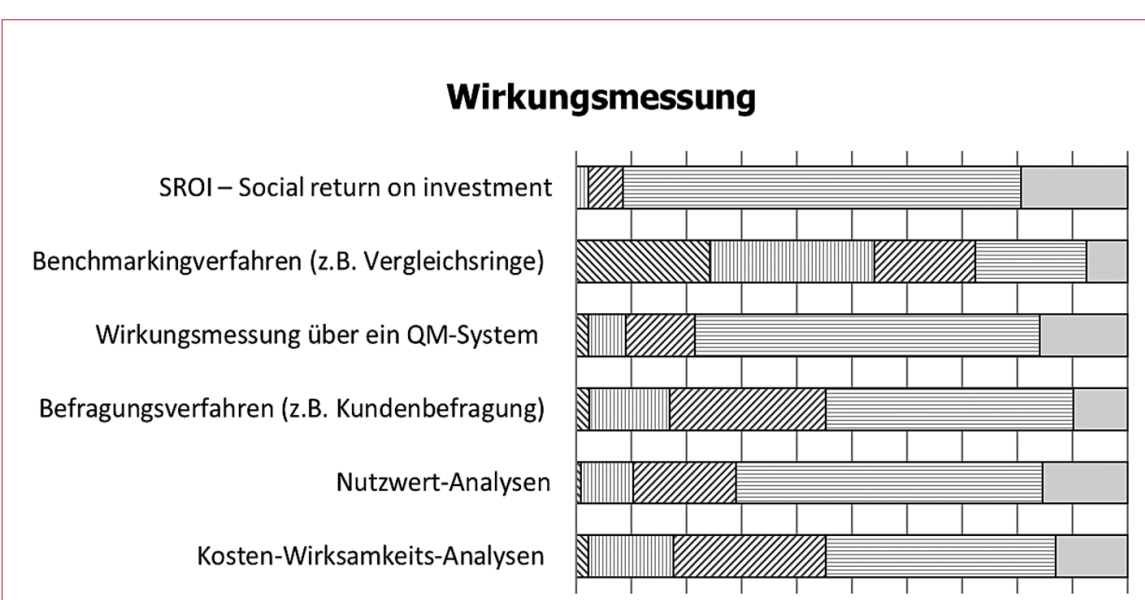

0\% 10\% 20\% 30\% 40\% 50\% 60\% 70\% 80\% 90\% $100 \%$

\$umfassend vorhanden $\square$ eher vorhanden $\square$ wenig vorhanden 目 nicht vorhanden $\square$ k.A.

Abb. 4: Verfahren zur Wirkungsmessung sind bei kommunalen Jugend- und Sozialhilfeverwaltungen kaum vorhanden. 
Sozialhilfe nur 60 Prozent Fachcontrolling haben (vgl. Abb. 5 und 6).

In ähnlich vielen Fachbereichen in der Jugend- und Sozialhilfe gibt es teilweise Controlling. Einem Viertel der Teilnehmenden aus der Sozialhilfe stehen dafür über die Hälfte der Fachbereiche in der Jugendhilfe gegenüber, die die Frage nach dem Vorhandensein von Fachcontrolling mit »ja « beantworten.

Dieser deutliche Unterschied ist möglicherweise mit dem stärkeren Entwicklungsanspruch in der Jugendhilfe zu erklären. In den befragten Fachbereichen werden die Daten aus dem Fachcontrolling zu nahezu gleichen Teilen aggregiert, nicht aggregiert und teilweise aggregiert.

In der Untersuchung wurde die große Bedeutung der Hilfeplanung für das Fachcontrolling deutlich. Die in den Hilfeplänen formulierten Ziele können im Controlling aggregiert werden und Hinweise über die Wirksamkeit von Maßnahmen und Trägern geben. Im Fall, dass Daten zusammenfassend ausgewertet werden, können die ermittelten Ergebnisse zur Strategieplanung genutzt werden und entsprechend Einfluss nehmen.

In nur einem kleinen Teil der Fachbereiche fließen die ermittelten Ergebnisse aus dem Fachcontrolling in die Strategieplanung mit ein. Im größten Teil der Fachbereiche ist dies teilweise der Fall und über ein Viertel antwortet auf die Frage danach mit »nein $«$.

\section{Vorhandensein von Fachcontrolling in der Jugendhilfe}

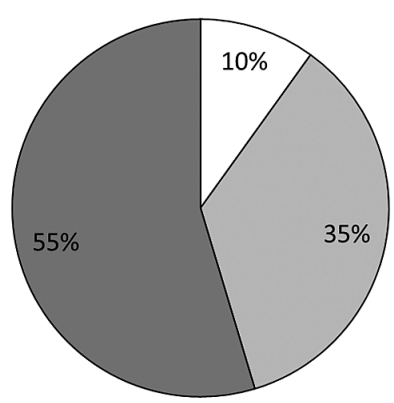

$\square$ nein

$\square$ teilweise

$\square$ ja

Abb. 5: Die kommunalen Jugendhilfeverwaltungen setzen das Fachcontrolling in starkem Maße ein.

\section{Zusammenfassung}

Zusammenfassend kann festgestellt werden, dass sich Controlling in Sozial- und Jugendhilfeverwaltungen in vielen Kommunen noch in einer konzeptionellen Aufbauphase befindet.

Die Einführung und Umsetzung von Controlling sehen Mitarbeitende und Führungskräfte häufig mit einer gewissen Skepsis. Gleichwohl steht Controlling vor der Aufgabe, sie am Controllingprozess zu beteiligen, glaubwürdig mit erforderlichen Daten zu versorgen und ergebnisbezogene Kommunikationsprozesse zu gestalten.

Der Controller oder die Controllerin braucht für die erfolgreiche Controllingeinführung und Controllingent-

\section{Vorhandensein von Fachcontrolling in der Sozialhilfe}

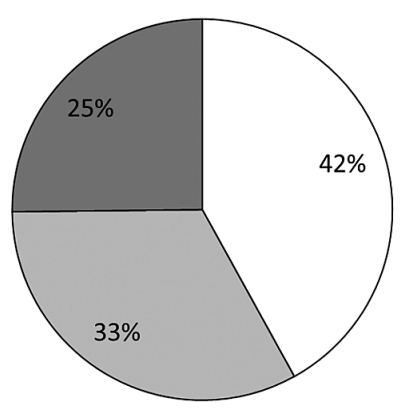

$\square$ nein

$\square$ teilweise

$\square$ ja

Abb. 6: Bei den kommunalen Sozialhilfeverwaltungen ist das Fachcontrolling noch ausbaufähig.

Die fehlende Verbindung von Fachcontrolling und Strategieplanung folgt den Ergebnissen zur strategischen Steuerung. Wo strategische Steuerung nicht nachhaltig etabliert ist, fehlt die Verbindung zu qualitativen Fragestellungen. wicklung den unbedingten Rückhalt der obersten Leitung. Er oder sie hat die Aufgabe, den Nutzen von Controlling zu kommunizieren und hinter für die Anwendung erforderlichen Controllingprozessen zu stehen.
Ein Teil des Einführungsprozesses ist die Analyse interner- und externer Adressaten, die Erwartungen an das Controlling richten. Mit ihnen ist zu klären, welcher Informationsbedarf besteht und wie Controlling mit den Adressaten aktiv kommuniziert bzw. wie ein entsprechendes Berichtswesen zu gestalten ist. Das subjektiv geäußerte Informationsbedürfnis ist anzureichern durch objektiv erforderliche Informationen für die Steuerung der jeweiligen Organisation. Fachliche und den Auftrag betreffende inhaltliche Kriterien sind dazu heranzuziehen. 